\title{
FAULT DETECTION OF FLIGHT CRITICAL SYSTEMS
}

\author{
Jorge L. Aravena, Louisiana State University, Baton Rouge, LA \\ Fahmida N. Chowdhury, University of Louisiana, Lafayette, LA
}

\begin{abstract}
This paper describes initial results of a project developing fault tolerant control systems for critical aircraft systems and focuses on the early detection of faulty components. The main goal is to present the use of signal processing techniques to analyze sensor information and data-mine changes that could be attributable to faulty behavior. The approach compares well with residual-based techniques but requires only output data. Hence, it could be applied to situations where residual-based approaches are not feasible.

In this paper we present the use of orthogonal filter banks as the processing elements to create fault indicators. The case study is an F14 jet fighter. Using computer simulations we create various faults and monitor the plane angle of attack. We show that the filter bank creates a number of orthogonal components, some of which have a clearly distinct pre and post fault behavior. Moreover, this behavior changes with the type of fault suggesting that it is possible to classify the faults.

Another significant issue addressed in this presentation is the generation of alarm signals based on the results of the fault detector. The paper discusses how the information in the components can be processed to create automatic alarm systems.
\end{abstract}

\section{Introduction}

The work described here is being developed as part of the research project Aircraft Safety: Control Upset Management, sponsored by NASA under the EPSCoR 2001 program, and whose goal is to improve aircraft safety. The project involves Louisiana State University, Baton Rouge, University of Louisiana at Lafayette and The University of New Orleans. In order to place this work in a proper perspective it is necessary to provide a brief overview of the complete project. The diagram in Figure 1 highlights the three basic components of this project: (1) Virtual Instrumentation, which provides tools for sensor fault detection, increases the reliability of critical instruments and may generate "virtual sensor readings" in the event of failures; (2) Fault Early Warning, which uses sensor readings to detect the onset of a component failure with sufficient time to take corrective actions; and (3) Fault Tolerant Control, which uses innovative concepts to create control systems that can operate safely even under conditions that would cause loss of control in conventional implementations .

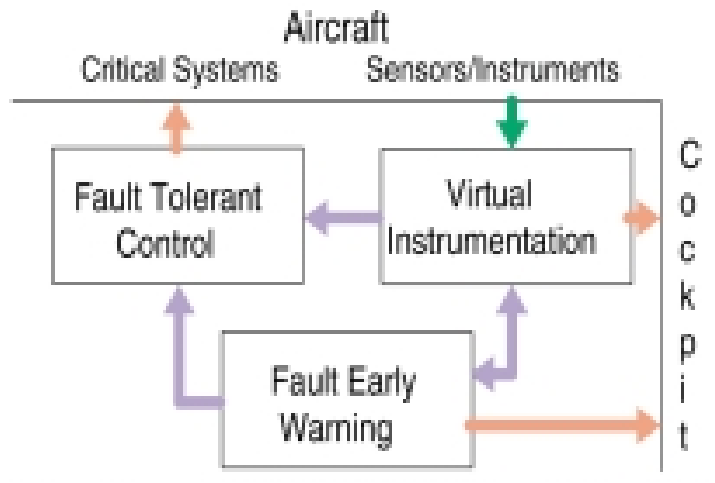

\section{Figure 1: Conceptual overview of the complete research project}

Generation of reliable readings is a goal of Virtual Instrumentation. The readings are essential to verify the status of the plane. Under full instrumentation, an anomaly must affect some sensors. The effect may be quite small at the onset of a fault but can be enhanced with suitable signal processing techniques. This enhancement is developed by the Fault Early Warning component. These two activities can be closely related but use different tools to achieve their goals. On the other hand, early fault detection can also be interpreted as "extracting knowledge from large amounts of data." In this case one adds a flavor of data mining to the problem [1].

In a significant enhancement over current aircraft control systems, both activities are integrated to improve the effectiveness of control 
systems. Information on sensor and component faults is incorporated in the design of Fault Tolerant Controllers in a new control paradigm called Generalized Internal Model Control.

The work described here belongs to the Fault Early Warning component of the project. The basic premise underlying this work is one of observability of faults. The information about the faulty behavior must be present in the sensors being used but it may be hidden, especially at the onset of a fault. If that early information can be ferreted out it may be possible to provide early detection.

There is a significant amount of work on residual-based fault detection techniques. The literature on this subject is too numerous to review here. We cite only some interesting survey works ([2], [3]) and relevant works focusing mostly on avionics subjects ([4]-[8]). Our intention is to create an alternative fault detection paradigm that can operate in situations where residual-based methods cannot be applied, and that can also take advantage of its positive aspects when it can be used. The technique was initially suggested for power systems applications ([9]) where the essentially sinusoidal regime makes it attractive.

The approach described in this work consists of the following steps: (1) Enhancing effect of faults on sensor reading; (2) Creating indicators that can be used to test for the presence of faulty behavior.

\section{Enhancing the Effect of Faults on Sensor Readings}

Residuals can, in fact, be considered an example of enhancement of the effect of faults. In this case the "normal behavior," obtained from a model of the system, is subtracted from the readings isolating the deviations attributable to faults. The case of interest to us here is that when such a model does not exist, and cannot be created on line due, for example, to the lack of information on system inputs.

Detectable faults, especially progressive faults will affect sensor readings. However, the effect on sensors may be quite small at the onset of a fault. The following simulation example illustrates this point. The mathematical models is given by the differential equation

$$
\tau \frac{d \boldsymbol{h}}{d t}+(1+K) h-\lambda(t) h=q(t)
$$

The function $\lambda(t)$ is a ramp function starting at $\mathrm{t}=20$. The model was built to emulate a tank with a level control system, subject to a sinusoidal input and with a progressive leak starting at $\mathrm{t}=20$ that, eventually, makes the control system go unstable. Figure 2 shows the sensor reading of the output of the system. The changes in amplitude are apparent towards the end of the simulation but it is not easy to determine the onset of the fault $(\mathrm{t}=20 \mathrm{~s})$.

One technique to enhance changes in the sensor readings, and that will be described below, decomposes the reading into orthogonal components. Figure 3 displays the output of a filter bank decomposing the reading in four components. Three "detail" components show a drastic transient at the onset of the fault.

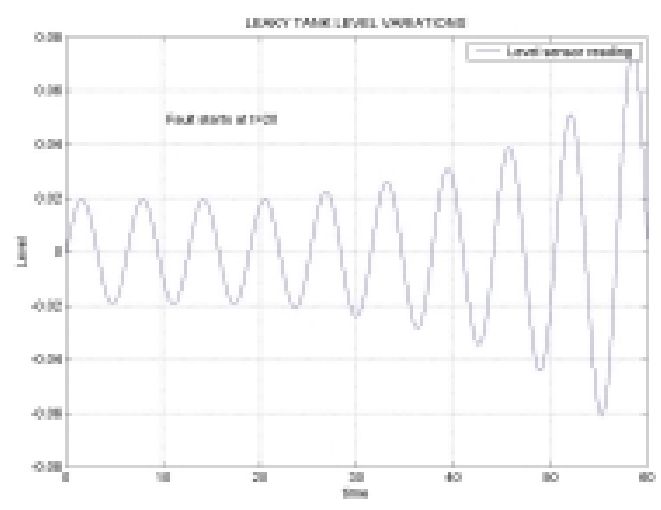

Figure 2:Sensor reading of a system undergoing a progressive fault

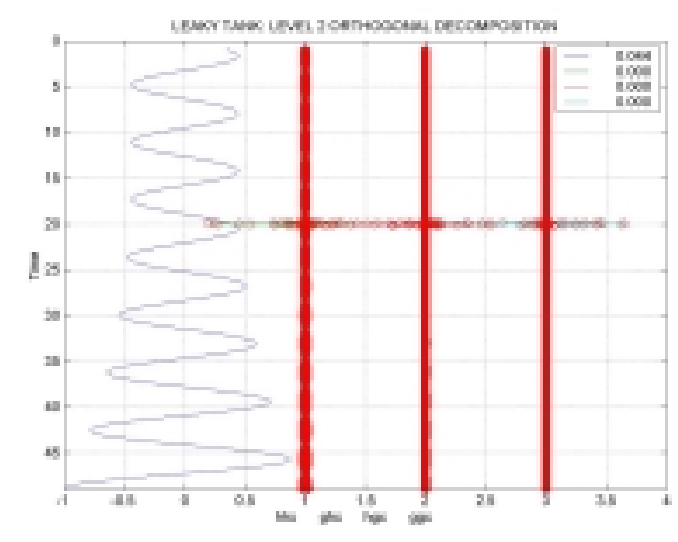

Figure 3: Decomposition of sensor reading to enhance changes . 
An essential feature is that the processing is carried out only on the sensor readings. Since it does not require the availability of inputs and outputs, technically it can be viewed as a modelfree detection approach.

\section{Filter Bank Orthogonal Decomposition}

This approach for enhancing the effect of changes is based on the premise that such changes will be small details in the complete signal. Hence they may not be apparent in a low-resolution view of the signal. However, if one can isolate details of the signal, the changes will appear as significant components in some detail views. In a sense, the low resolution, or background view is a noise when the goal is the determination of changes and the purpose of the enhancement is to increase the detail-to-background ratio.

Wavelet based filter banks offer a convenient implementation for a multiresolution signal representation. In the conventional approach, a signal is decomposed in a low-resolution component and a detail component. The lowresolution component can undergo further decompositions while the detail signal is not decomposed again, creating a not symmetrical decomposition. In order to increase the sensitivity to details we have developed a symmetrical decomposition. The block diagram for the case of four levels is shown in Figure 4

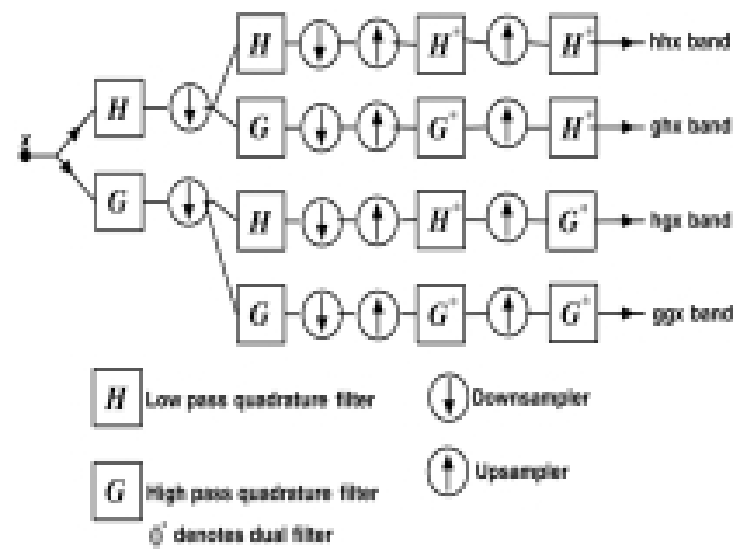

Figure 4: Basic filter bank structure
The filter, $H$, is low pass and its application creates low-resolution views. The filter, $G$, is high pass and creates signal details. For these examples both filters were obtained form Daubechies' wavelets ([10]) and are of length 16.

Many experiments were performed in order to test the ability of the filter bank decomposition to enhance the effect of faults on sensor readings. The next results show a representative example. Using a public domain model for an F14 plane, we implemented a sudden change in the time constant of the actuator moving the aileron. The stick was driven with a colored, band limited, random signal to emulate a demanding scenario. Monitored sensor reading is the measurement of the plane angle of attack. Figure 5 shows the angle of attack of the fault free model overlaid with the faulty case. The purpose of this display is to illustrate the small effect of the fault and the potential sensitivity that can be obtained with this type of enhancement.
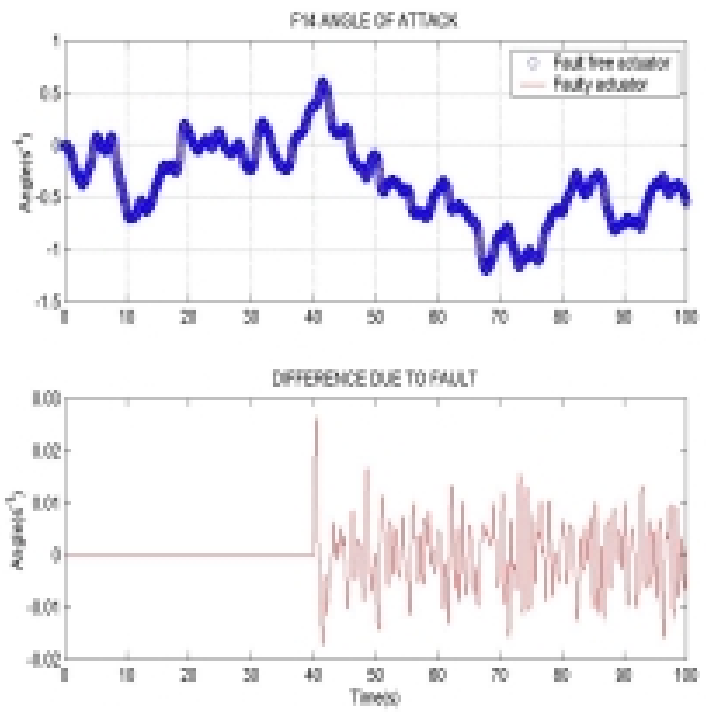

\section{Figure 5: Effect of the fault on the sensor reading}

The next figures shows the eight outputs of a filter bank when the input is the sensor reading (Figure 6) and when the input is the G-force sensor (Figure 7), both for the faulted case. Figures $8 \mathrm{a}$ and $8 \mathrm{~b}$ show the decompositions of the same variables for a different fault. In this case a faulty filter in the controller. Each component is normalized to a maximum of one to enhance clarity of the display. Some of the values may not be quite realistic due to the randomness of the stick movements but they 
serve well to illustrate the concept. It can be easily seen that several components show a distinct pre and post fault behavior. This result supports the concept of the filter bank as a valid procedure to enhance the effect of faults on sensor readings. Moreover, different components show different sensitivity to the angle of attack than to the g-force readings (e.g., components number 3,6 and 9)

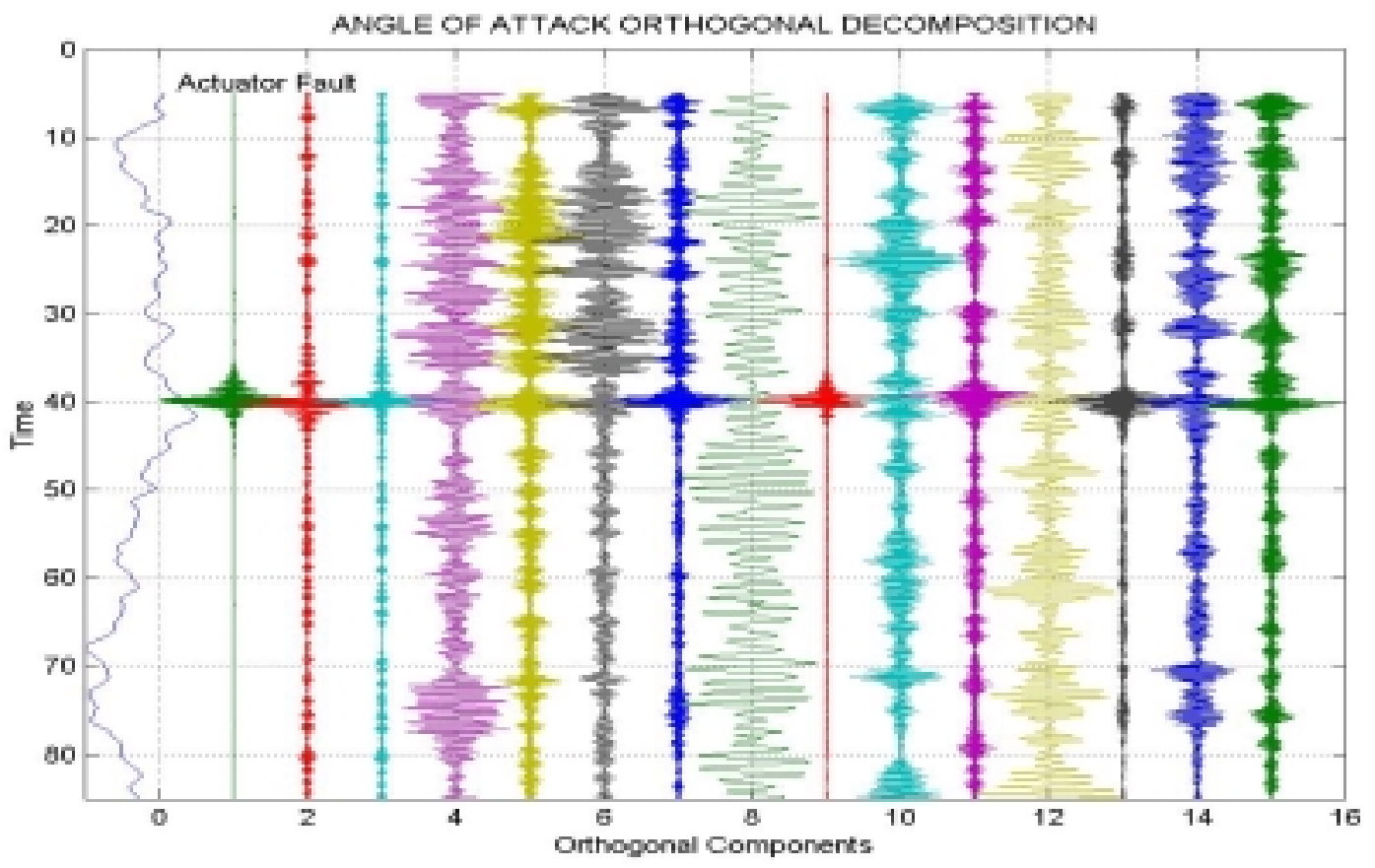

Figure 6: Outputs of Filter Bank for the case of angle of attack. Actuator fault at $t=40$. Signals are normalized for enhanced clarity.

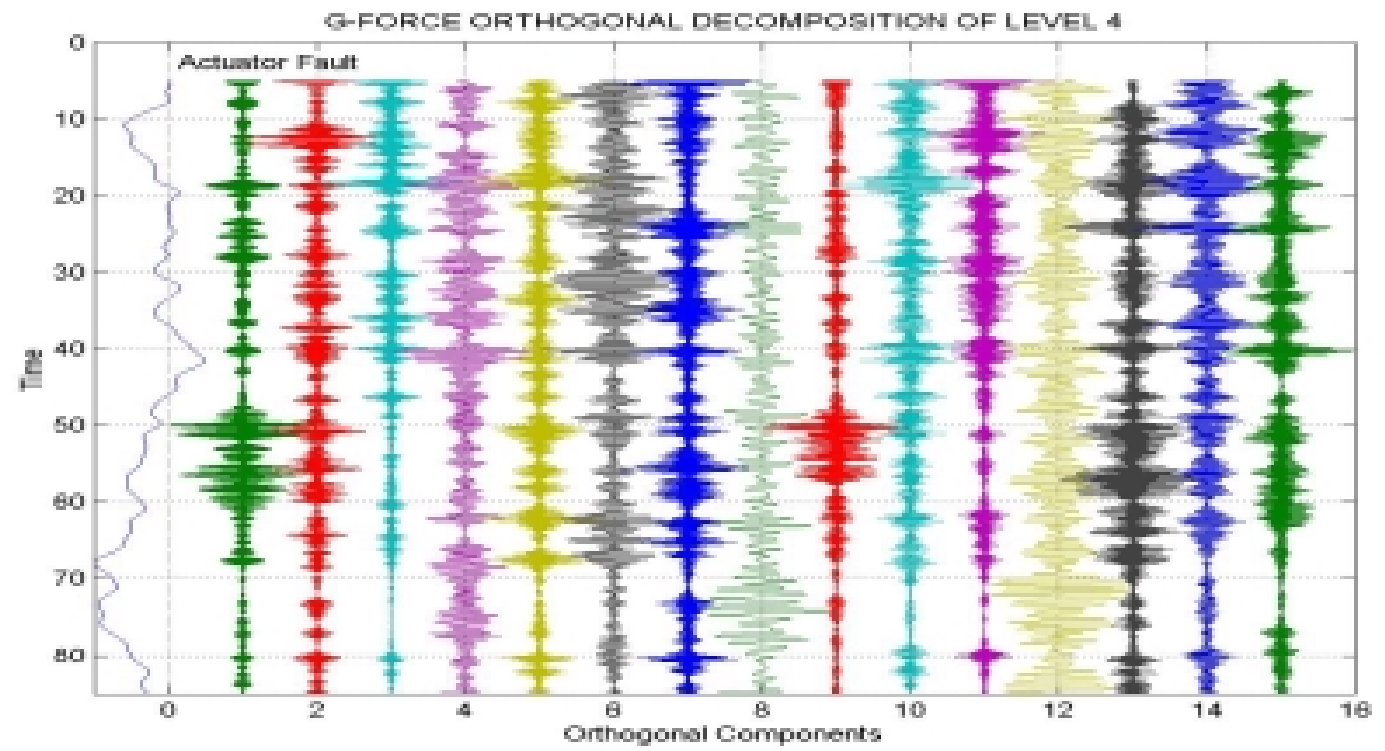

Figure 7: Output of filter bank when processing G-force readings. Actuator fault at $t=40$ 


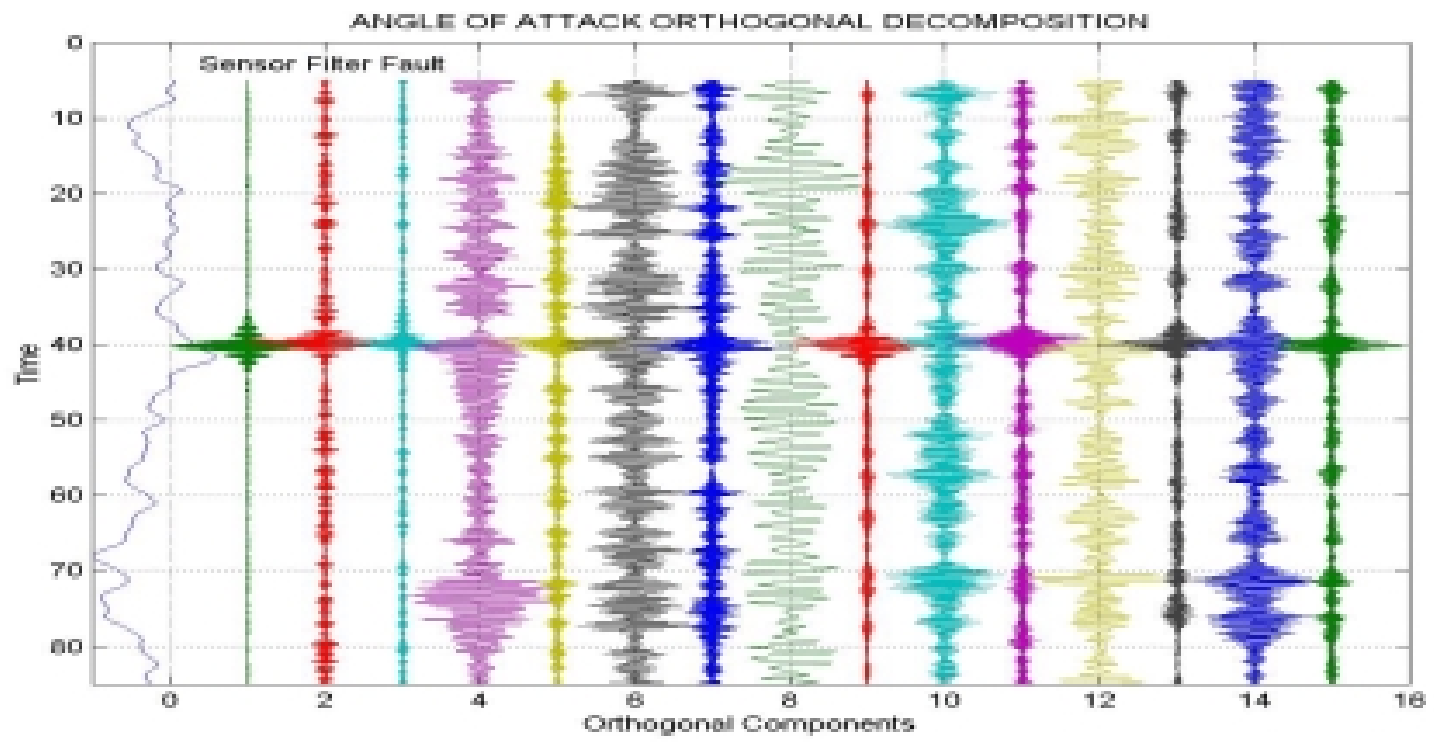

Figure 8a: Angle of attack decomposition for fault in controller filter at $\mathbf{t}=\mathbf{4 0}$

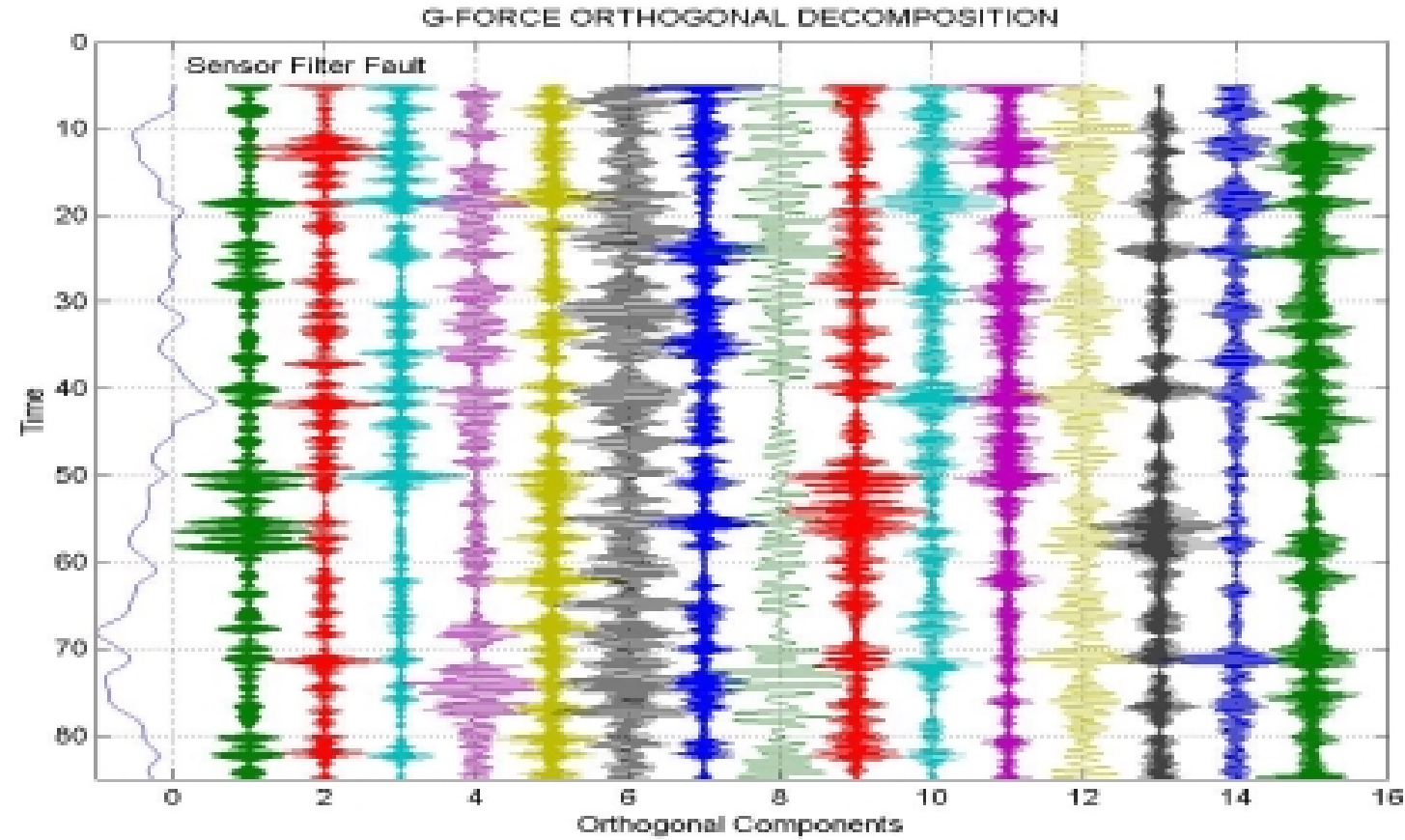

Figure 8b: G-force decomposition for fault in controller filter at $\mathrm{t}=\mathbf{4 0}$

These basic experiments also support the potential of the filter bank decomposition to permit fault discrimination. They also suggest that techniques of sensor fusion and data mining might be used to advantage. 
It is necessary to gain understanding of the operation of the filter bank in order to develop additional sensor enhancing techniques. The bank is based on quadrature mirror filters. Ideally the low pass digital filter, $H$, has normalized bandwidth $[O$ $\pi / 2]$ while the high pass filter, $G$, has a bandwidth $\left[\begin{array}{ll}\pi / 2 & \pi\end{array}\right]$. The operations of downsampling and upsampling eliminate redundancy and, essentially, each band describes the behavior of the signal in a narrow frequency band. The following table is the building block to determine the frequency ranges contained in each band

\section{Table 1. Normalized Frequency Ranges for Decomposition in Four Bands}

\begin{tabular}{l|l|} 
Processing & Frequency Range \\
HH & $0-\pi / 4$ \\
GH & $\pi / 4-\pi / 2$ \\
GG & $\pi / 2-3 \pi / 4$ \\
HG & $3 \pi / 4-\pi$ \\
\hline
\end{tabular}

The code assigned to each band retains the processing done and can be used to estimate the frequency band covered by a given component. For example, the component labeled ghhhx ideally contains the information in the frequency range [ $\pi / 16 \pi$ /8].

The difference in pre and post fault behavior for a given band is attributed to the variation in frequency content due to the fault. The changes are very small and can be easily lost in the complete signal. Only when the details are isolated, the changes in content become apparent. The interpretation suggests that focusing on the energy distribution as a function of the frequency (spectral energy density) may be a viable approach to detect small changes due to faults. This approach is considered in the next section

\section{Frequency Domain Enhancement}

The short time Fourier transform is a logical initial choice to study spectral energy distribution as a function of time. It is apparent that, in order to increase sensitivity, one must focus on the high resolution details of a signal. The structure of this enhancement technique is displayed in Figure 9 below. The low resolution generator has been implemented with a wavelet based filter bank and is in fact the lowest resolution component of the previously used filter bank. Other alternatives are currently under investigation but it should be noted that the low resolution generator could be replaced by a nominal model and the spectral analysis applied to the residuals.

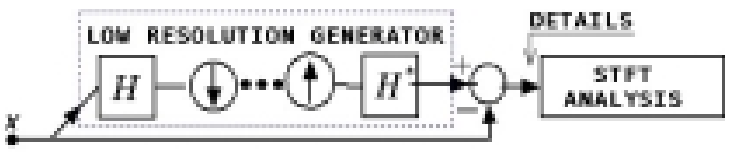

\section{Figure 9. Basic structure for frequency domain enhancement}

Figure 10, below, displays the application of the STFT enhancer to the G-force variable.

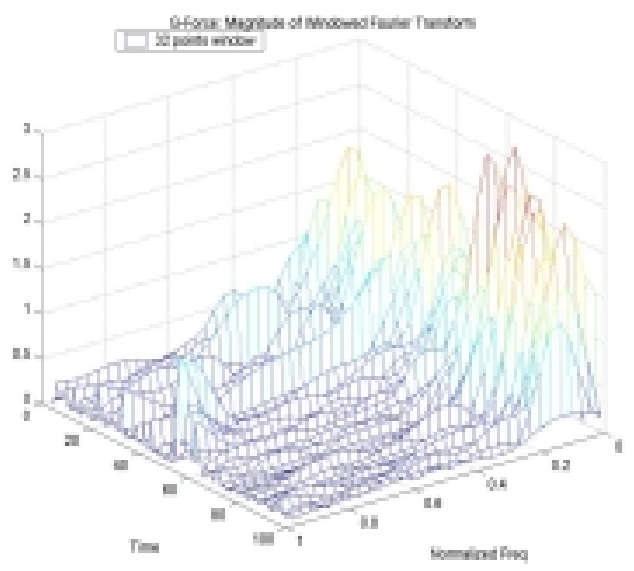

Figure 10. STFT enhancing of G-force details

Results obtained with this enhancer are not conclusive. There are differences in the spectral energy distribution pre and post fault behaviors. 
However, the changes do not appear clear. Similar analysis performed on the angle of attack reading support the conclusion. We attribute this lack of discrimination capability to two basic reasons: (1) Dumping the details in a single signal; (2) the fixed length of the window in the short time Fourier Transform.

The filter bank decomposition separates the details into several components. Only some components are sensitive the faults. Hence, identifying and concentrating on those bands improves the detail-to-background ratio. The limitation of the fixed window length can be overcome with multi-resolution decompositions such as the continuous wavelet transform. However, the amount of computations can be quite extensive.

We present here a procedure that is currently under development and that appears to solve both perceived limitations.

\section{Discrete Scale-Frequency Distribution}

We show here a procedure, based on the continuous wavelet transform that permits the reduction of computational complexity and, at the same time, retains the multi-resolution characteristic of the wavelet transform. In order to improve clarity of the presentation we start by establishing the notation used.

The continuous wavelet transform (CWT) of a signal, $x(t)$, is defined for $a>0$, and all values of the time variable, $b$, by the integral

$$
\boldsymbol{C}_{\boldsymbol{x}}^{\psi}(\boldsymbol{a}, \boldsymbol{b})={ }_{-\infty}^{\infty} \boldsymbol{x}(\boldsymbol{t}) \psi_{\boldsymbol{a}}(\boldsymbol{t}-\boldsymbol{b})^{*} d t
$$

where

$$
\psi_{a}(t)=\frac{1}{\sqrt{a}} \psi\left(\frac{t}{a}\right)
$$

is the time scaled copy of the basic wavelet.

It is known that the CWT is highly redundant and that one can use a discrete set of scales to obtain non-redundant decompositions. The best known of these non-redundant decompositions is the multi-resolution representation using a dyadic scale and valid only for some wavelets. That decomposition leads to the filter banks used previously.

Formulating the transform in the frequency domain one can establish general conditions to select discrete sets of scale that permit the exact reconstruction of a signal. For this, let

$$
\boldsymbol{C}_{\boldsymbol{x}}^{\Psi}(\boldsymbol{a}, \boldsymbol{\omega})=\boldsymbol{F}\left\{\boldsymbol{C}_{\boldsymbol{x}}^{\psi}(\boldsymbol{a}, \boldsymbol{b})\right\}
$$

be the Fourier transform of the CWT, with respect to the time variable. One can show

$$
C_{x}^{\Psi}(a, \omega)=X(\omega) \sqrt{a} \Psi(a \omega)
$$

with $\Psi(\omega)$ being the Fourier transform of the basic wavelet.

Assume now that a finite set of scale $\left\{\boldsymbol{a}_{1}, \boldsymbol{a}_{2}, \ldots, \boldsymbol{a}_{\boldsymbol{M}}\right\}$ is selected and the wavelet transform is computed at only those scales. That is to say one has available the collection

$$
\left\{\boldsymbol{C}_{\boldsymbol{x}}^{\Psi}\left(\boldsymbol{a}_{\boldsymbol{k}}, \omega\right), \boldsymbol{k}=1,2, \ldots \boldsymbol{m}\right\} \text {. }
$$

It is possible to show that the signal, $x(t)$,can be perfectly reconstructed from the collection of transform samples if and only if the wavelet is

admissible and $\lambda(\omega)={ }_{k} \frac{\left|\Psi\left(a_{k} \omega\right)\right|^{2}}{a_{k}}$ is non zero on the support of $X(\omega)$. This result is intuitive. As long as the wavelets 'cover the spectrum of the signal', one can reconstruct it from the collection $\left\{\boldsymbol{C}_{\boldsymbol{x}}^{\Psi}\left(\boldsymbol{a}_{\boldsymbol{k}}, \boldsymbol{\omega}\right), \boldsymbol{k}=1,2, \ldots \boldsymbol{m}\right\}$.

And each term can be considered to give a description of the signal at the resolution defined by the value of the corresponding scale. From a filtering point of view, each term is the output of a filter with frequency response $\sqrt{a_{k}} \Psi\left(a_{k} \omega\right)$. Since the wavelet is admissible, it must have a band pass characteristic. The value of the scale will dilate or compress the pass band. Figure 11 illustrates this concept using an ideal band-pass characteristic 


\section{Idealized Wavelet}

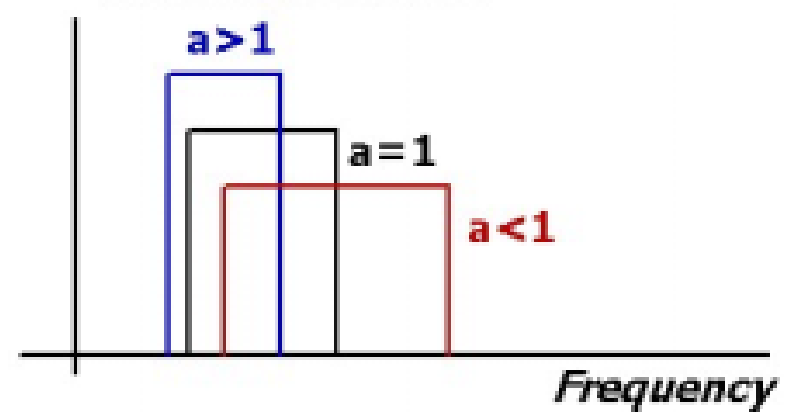

Figure 11. Effect of the scale on a wavelet

Increasing the scale narrows the band and moves it to lower frequencies. Hence one gets lower resolution views of the signal. In a similar way, decreasing the scale will produce views containing higher frequency components associated with finer details.

Issues currently under investigation include:

(1) The type of wavelet to be used and the selection of scales; and (2) the technique to create details from the multiresolution representations. A conceptually simple approach is to consider the outputs of the filters as signals arranged in decreasing scales and apply an orthogonalization algorithm on the filter outputs. Based on the figure is it assumed that each new orthogonal component will contain higher frequency details than the previous one, creating a situation similar to that of the orthogonal filter bank. The main difference being the fact that the center frequencies of each filter is completely under user control and it should be possible to manipulate the amount of details in each component. The down side of this flexibility is that the resulting filters are complex and not easy to implement.

\section{Creating Automatic Fault Detectors}

Results in the previous section support the fact that it possible to enhance the effect of faulty behavior on sensor reading. The filter bank decomposition creates components that show distinct pre and post fault behavior. The issue that we address in this section is the automatic detection of the changes in these components. The solution that is being developed creates instantaneous patterns and classifies these patterns. As a proof of concept we show here results using a 2-D pattern created using components 5 and 7 of the filter bank decomposition of the angle of attack for the actuator fault.

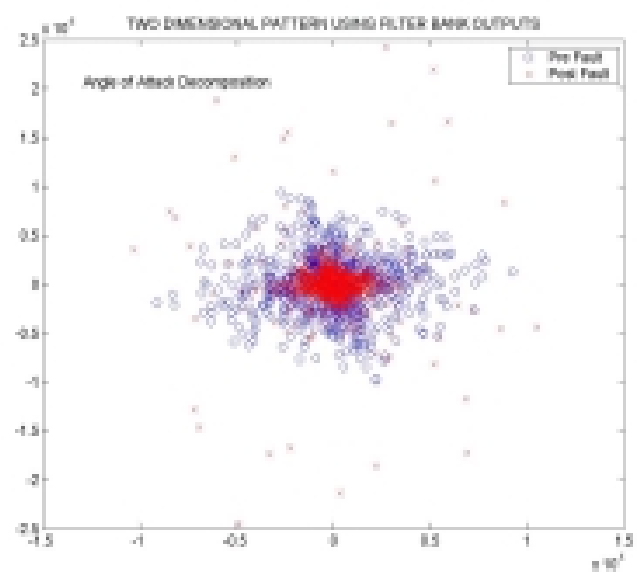

Figure 12. 2-Dimensioanl patterns based on filter bank outputs

The pre and post fault patterns show a clearly different behavior. But a simple linear classifier does not appear as a viable solution.

Figure 13 shows an alternative detector using the norm of the instantaneous values constructed using components 5,7, and 15 of the filter bank decomposition.

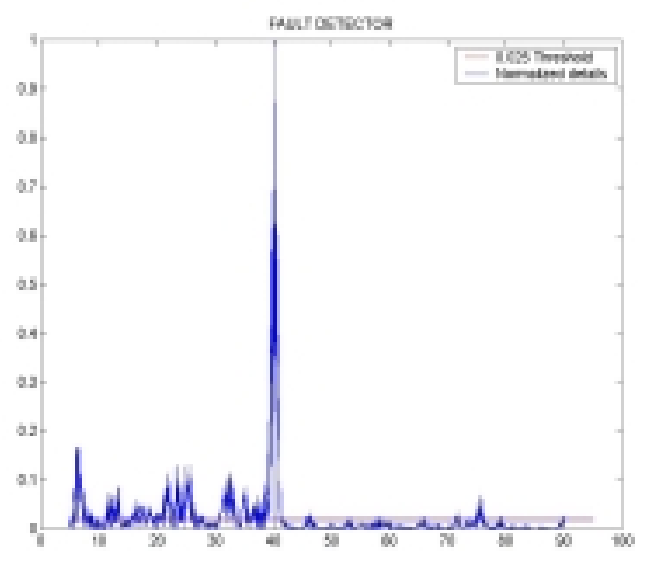

Figure 12. A simple fault detector based on filter bank outputs

This detector shows a clear difference between pre and post fault behavior. But it also shows the possibility of false alarms that need to be examined in more detail. 


\section{Conclusions}

This paper reports preliminary results on an alternative approach to fault detection. We advocate the use of signal processing techniques to analyze sensor data and detect changes that can be attributed to faulty behavior. The approach does not require the existence of mathematical models and can be applied to situations where residual-based techniques cannot be applied. Experiments with noise-free data collected from computer simulations show that filter bank processing can create detail signals with distinct pre and post fault behavior. Moreover, the outputs of the filter bank can be processed to create automated fault detectors.

\section{References}

[1] J. Han, M. Kamber, "Data Mining: Concepts and Techniques," Academic Press, 2001

[2] J. Gertler, " Survey of model-based failure detection and isolation in complex plants," IEEE Control System Magazine, Vol. 8, pp. 8-11, 1988.

[3] P. M. Frank, “Fault diagnosis in dynamic systems using analytical and knowledge-based redundancy -- a survey and some new results," Automatica, Vol. 26, No. 3, pp. 459-474, 1990.

[4]X. Pei and F. N. Chowdhury, "Unsupervised neural networks for fault detection and classification in dynamic systems", Proc. IEEE International Conference on Control Applications (CCA99), (640 - 645), Aug. 1999

[5] R. C. Montgomery and A. K. Caglayan, "Failure accomodation in digital flight control systems by Bayesian decision theory", AIAA Journal of Aircraft, Vol. 13, No. 2, Feb. 1976

[6] R. C. Montgomery and D. B. Price, "Failure accommodation in digital flight control systems accounting for nonlinear aircraft dynamics", AIAA Journal of Aircraft, Vol. 13, No. 2, Feb. 1976

[7] B. Weinstein, "Detecting controller malfunctions in electromagnetic environments: part I - modeling and estimation of nominal system function", Proc. IEEE Int. Conf. on Contr. Appl., (1525-1530), Aug. 1999

[8] C. M. Belcastro, “Detecting controller malfunctions in electromagnetic environments: part
II - design \& analysis of the detector", Proc. IEEE Int. Conf. on Contr. Appl., (1531-1537), Aug. 1999

[9] F.N. Chowdhury, and J.L. Aravena, “A Modular Methodology for Fast Fault Detection and Classification in Power Systems," IEEE Trans. Contrl. Syst.Techn., pp 623-632, Vol. 6, No. 5, Sep. 1998.

[10] I. Daubechies, Ten Lectures on Wavelets, CBMS-NSF Regional Conference Series in Applied Mathematics, SIAM 1992 